\title{
Maternal/Child Social Support and Food Security in Relation to Child Height and Bmi in Four Low- and Middle- Income Countries: Mediation Analysis of Young Lives Data
}

\author{
Hwa-Young Lee \\ Harvard University T H Chan School of Public Health https://orcid.org/0000-0003-2591-1436 \\ In Han Song ( $\square$ isong@yonsei.ac.kr) \\ Yonsei University \\ Ichiro Kawachi \\ Harvard University T H Chan School of Public Health
}

\section{Research}

Keywords: social support, child height, child BMI, mediation analysis, low-and middle-income country

Posted Date: May 27th, 2021

DOl: https://doi.org/10.21203/rs.3.rs-520037/v1

License: (c) (i) This work is licensed under a Creative Commons Attribution 4.0 International License.

Read Full License 


\section{Maternal/child social support and food security in relation to child height \\ 2 and BMI in four low- and middle- income countries: Mediation analysis \\ 3 \\ of Young Lives data}

4

5

6 Affiliations:

7

(1)
Hwa-Young Lee ${ }^{1,2}$, In Han Song ${ }^{2,3}$, Ichiro Kawachi ${ }^{4}$.

1. Department of Global Health and Population, Harvard T.H.Chan School of Public Health, Boston, MA, USA

2. Institute of Convergence Science (ICONS), Convergence Science Academy, Yonsei University, Seoul, The Republic of Korea

3. Graduate School of Social Welfare, Yonsei University, Seoul, The Republic of Korea

4. Department of Social and Behavioral Sciences, Harvard T.H. Chan School of Public Health, Boston, MA, USA

Email address:

Hwa-Young Lee: diana0224@gmail.com; hwlee@hsps.harvard.edu.

In Han Song: isong@yonsei.ac.kr

Ichiro Kawachi: ikawachi@hsph.harvard.edu

Graduate School of Social Welfare, Yonsei University, Seoul, The Republic of Korea 50 Yonsei-ro Seodaemun-gu, Seoul, 03722, Korea 


\section{Maternal/child social support and food security in relation to child height and BMI in four low- and middle- income countries: Mediation analysis of Young Lives data}

32 Abstract

\section{Background}

34 Poor nutritional status in childhood is associated with an elevated risk of mortality and morbidity 35 later in life. Previous studies showed a positive association between specific types of social capital 36 and child nutritional status. Our study examined whether improved food security mediates the

37 impact of maternal and child social support on child height and body mass index (BMI) in four 38 low- and middle-income countries.

\section{Methods}

40 We used data from the Young Lives cohort study comprising roughly 1,000 children at age 8 41 and 12 in Vietnam, Ethiopia, India, and Peru. Outcome variables were z-score for height (HAZ) 42 and BMI (BAZ).

\section{Results}

44 Belonging to the top half of maternal financial support and child financial support was positively 45 associated with child HAZ at age 12 in Peru. Belonging to top half of overall maternal support 46 among children aged 8 in Vietnam, and maternal financial support among children aged 12 in 47 India were also positively associated with child BAZ. A positive association of food security was 48 only found with maternal financial support among children aged 12 in Peru. However, food 49 security did not play a significant role in mediating the effect of maternal financial support on 50 child HAZ at age 12. 


\section{Conclusions}

52 Strengthening social support to improve child nutritional status may not be a sufficient

53 intervention in resource-poor settings because sources of supports may lack sufficient food

54 resources to share. Considering between-country heterogeneity, a "one size fits all" approach for

55 enhancing social capital may not be appropriate.

56 Key words; social support, child height, child BMI, mediation analysis, low-and middle-income 57 country.

58

59

60

61

62

63

64

65

66

67

68

69

70 


\section{Introduction}

Poor nutritional status in childhood has been linked to elevated risk of mortality and

73 morbidity later in life. ${ }^{1}$ Not only does impaired physical growth hamper child development

74 (defined as the attainment of gross motor and fine motor skills), psychosocial competencies, and

75 cognitive abilities, ${ }^{23}$ it also raises a risk of infectious disease. ${ }^{4}$ However, the worldwide prevalence

76 of child anthropometric failure including stunting, underweight, and wasting remain stubbornly

77 high and are concentrated in Low and Middle-Income Countries (LMICs). In 2019, 38\% and 34.5\%

78 of children aged 0-59 months in Eastern Africa and Oceania were estimated to have stunting

79 respectively, which is more than 15 times higher than in Northern America (2.6\%). ${ }^{5}$

According to the United Nations Children's Fund (UNICEF) framework of determinants

81 of child undernutrition, household food security is one of the important factors for child

82 undernutrition which is in turn affected by socioeconomic conditions and the national/global

83 context. $^{6}$ Food insecurity leads to inadequate dietary intake which affects height and weight

84 directly as well as indirectly by promoting disease occurrence. This was empirically demonstrated

85 in Humphries (2015) where children from chronically food-insecure households in Ethiopia, India,

86 Peru, and Vietnam had significantly lower Height for Age Z-score (HAZ) compared to

87 households that were consistently food-secure. ${ }^{7}$

Social capital, defined as the resources embedded within social networks, ${ }^{8}$ has been

89 demonstrated to positively affect health. Social capital can be analyzed as an individual attribute,

90 i.e., as an individual's access to social support within a network. Social capital can be analyzed

91 also as a property of the collective, e.g., norms of mutual assistance within a group. ${ }^{910}$ Although 
92 social capital has long been discussed in social sciences, the emergence of social capital in

93 development practice is relatively recent. ${ }^{11}$ Several studies have found a positive association

94 between maternal or household social capital and child nutritional status. ${ }^{12-15}$ However, it is

95 difficult to reach any definite conclusion because characteristics of the sample and measure of

96 social capital varied from study to study, and the results have been mixed.12-15

97 Studies that found a positive association between social capital and child nutritional status

98 suggested that increased food security may be the mechanism, whereby individuals share food

99 resources within their network or gain access to knowledge of where to obtain cheap sources of

100 food. A handful of studies have demonstrated an association between social capital and

101 household hunger or food security. ${ }^{16-18}$ However, most of these took place in high-income

102 countries (HICs) where food security is good on average, and therefore, there is an abundant

103 source of supports that food-insecure households can borrow food or receive food assistance from.

104 Only one study based in the LMIC setting has examined the role of social capital in the context of

105 a food support program within their community. ${ }^{19}$ Furthermore, none of studies examined the

106 mediating role of food security in the effect of social capital on child nutritional status in any

107 settings.

The results from these prior studies about association among maternal social capital, food

111 children are old enough to develop their own social networks or to participate in groups while

112 they are still in a growing phase, no studies have evaluated the association between child's social 
113 support and their nutritional status. Thus, our study aimed to examine 1) whether maternal and

114 child social support is associated with child height and Body Mass Index (BMI) 2) whether these 115 associations are mediated by food security.

\section{Methods}

\section{Study design}

Data were obtained from the older cohort of the Young Lives (YL) study, an international and longitudinal cohort survey performed in Ethiopia, India, Peru, and Vietnam. ${ }^{20}$ The older

121 cohort of the YL study comprises around 1,000 children aged 8 years when recruited in 2002 (wave

122 1). Subsequent data were collected at age 12 years in 2006 (wave 2), 15 years in 2009 (wave 3), 19

123 years in 2013 (round 4), and finally at age 22 years in 2016 (round 5). Data for our analysis were 124 extracted from waves 1 and 2.

127 oversampling of poor sites to serve the main study objective to explore the causes and 128 consequences of childhood poverty. ${ }^{20}$ For example, the most food-insecure areas encompassed the 129 sampling universe in Ethiopia. In Peru, the richest 5\% of districts were excluded from the sample.

130 However, final samples represent a range of regions, policy contexts, and living conditions. ${ }^{7}$ The

131 cohort in India consisted only of households from Andhra Pradesh while cohorts in the other 132 three countries were nationwide. At the second stage, all households with children of the right 133 age within the sites were listed, from which 100 households were randomly selected at each site. ${ }^{21}$ 
134 The response rate was above $90 \%$ in all the countries. Data were collected by a standardized, 135 interviewer-administered questionnaire from the child's main caregiver.

\section{Ethical review}

137 Approval for this study was granted by the Social Science Division of Oxford University, and 138 research ethics committees in Ethiopia, India, Vietnam, and Peru.

\section{Study indicators}

140 Child anthropometry

We assessed both child height and BMI, which is affected by chronic and acute nutritional

142 status respectively. ${ }^{22}$ Height was measured using stadiometers with standing plates and moveable

143 headboard which were locally made, and weight was measured by calibrated digital balance

144 (Soehnle). Height-for-Age Z score (HAZ) and BMI for -Age Z score (BAZ) were calculated using

145 the WHO 2007 standard. ${ }^{23}$ Staffs were adequately trained to measure anthropometries and 146 utilized techniques according to WHO guidelines. ${ }^{24} 25$ Extreme z-scores deemed biologically 147 implausible (<-6 and $>6$ for $\mathrm{HAZ}$, and $<-5$ and $>5$ for BAZ) were dropped according to the WHO 148 recommendation. ${ }^{26}$

149 Food security

150 Food security was asked differently in wave 1 and 2. In wave 1, respondents were asked 151 whether the household had gotten enough food to eat while in wave 2, they were asked whether 152 the household had experienced any food shortage in the last 12 months. "Yes" in wave 1 and "no" 153 in wave 2 was coded as one indicating that the household was food-secure. 

maternal social capital was measured while both maternal and child social support was measured

157 in wave 2. In wave 1, support received from groups in which the mother participated (support 158 from groups) as well as social support received from different types of individuals (support from 159 individuals) were combined into an index of maternal social support. For support from groups, 160 when the respondent answered that they belonged to any of seven different kinds of groups (trade 161 union, community association/co-op, women's group, political group, religious group, 162 credit/funeral group, and sports group), they were subsequently asked whether they had received 163 any support from that group. For support from individual, participants were asked whether they 164 had received support from any of nine different types of individuals (e.g., family, neighbors, 165 friends and so on). A total score of maternal social support was calculated by summing the 166 number of 'yes' resulting in a score range from 0 to 16, which were categorized by median split.

167 In wave 2, only financial support was examined for mothers while child social support was 168 examined comprehensively. Specifically, mothers were asked how many people they could rely 169 for material or financial support with seven response options (none, 1, 2, 3 5, 6 10, 11 15, 16 20, 170 12 30, and >30). Responses were then dichotomized into Yes (none) versus No (all others).

171 Children were asked whether there is someone who can help in six different types of situations 172 (detailed questions were described in Table S1). The overall level of child support was calculated 173 by summing positive responses resulting in a range of $0 \sim 6$, which were categorized by median 174 split. Some countries showed a skewed distribution of maternal and child social supports 
175 (presented in blue and red arrows in Fig 2.1 and Fig 2.3). We additionally examined financial

176 support for the child based on the question asking whether the child has someone who can help

177 when they needed pocket money (Table S1).

178 Covariates

179 Child characteristics included gender (female vs. male), birth order $\left(2^{\text {nd }}, 3^{\text {rd }}\right.$, and higher 180 than $4^{\text {th }}$ vs. $1^{\text {st }}$ ), and child's working status (yes vs. no). Caregiver factors included age in five-year 181 bands $(30 \geqq \&<35,35 \geqq \&<40$, and $40 \geqq$ vs. $<30)$, education level (completed vs. not completed 182 primary), and marital status (permanent partner vs. divorced, separated, single or widowed). 183 Household characteristics included household size (5 or 6 , and $>6$ vs. $\leqq 4$ ), residential location 184 (rural vs. urban), and wealth quintiles $\left(2^{\text {nd }}, 3^{\text {rd }}, 4^{\text {th }}\right.$ and $5^{\text {th }}$ vs. $\left.1^{\text {st }}\right)$. Wealth quintile was based on a 185 wealth index ranging from 0 to 1 which was calculated by averaging three variables: housing 186 quality, ownership of consumer durables, and access to services.

\section{Statistical analyses}

188 First, we summarized the distributions of maternal and child's social support, as well as HAZ and 189 BAZ according to maternal, child, and household characteristics for each of four countries. Then, 190 associations between level of maternal or child's social support and child's HAZ and BAZ at age

1918 and 12 were assessed using multivariable linear regression models. We introduced the 192 community cluster effect $\left(\varepsilon_{1}, \varepsilon_{2}\right.$, and $\left.\varepsilon_{3}\right)$ to the model using the 'cluster' option in 193 the STATA package. The model can be specified as follows;

$$
H A Z \text { or } B A Z=\alpha_{1}+\gamma \text { maternal or child social support }+\emptyset_{1} X_{1}+\varepsilon_{1} \text {, }
$$


Where $\mathrm{X} 1$ includes control variables except for food security, and $\varepsilon_{1}$ is a community random effect. support and child height and BMI, we fit a meditation model. We examined whether maternal or 200 child social support which showed a significant association with HAZ or BAZ in equation (1) is 201 associated with the probability of having food security, using the following reduced-form 202 specification.

208 HAZ or BAZ $=\alpha_{3}+\gamma^{\prime}$ maternal or child social support $+\sum_{i} \delta_{i}$ Food security ${ }_{i}+\emptyset_{3} X_{3}+\varepsilon_{3}-$ visualized as a mediation model proposed by Baron and Kenny (Fig 1) ${ }^{27}$. 

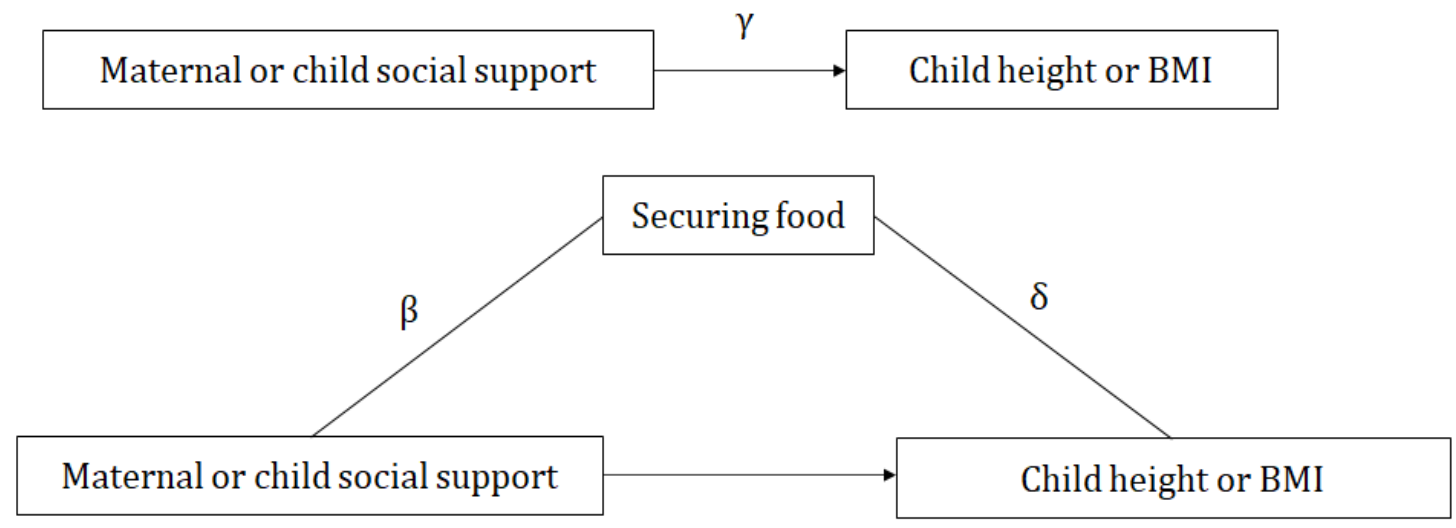

Figure 1. The mediation model. make a causal interpretation due to a potential omitted variable bias. To address these issues, we used the potential outcome framework introduced by Imai et al (2010) that use counterfactuals to identify causal effect and decomposes the total effect of a variable into direct and indirect (i.e.,

220 meditational) effect. ${ }^{28} 29$ In Imai et al 's model, the total effect is $\beta$ in the equation 1, which is a 221 total effect of social support on HAZ or BAZ (without food security effect). Average direct effect 222 (ADE) is the mean difference between two counterfactual states of initial conditions, assuming no 223 change in the mediator ( $\gamma^{\prime}$ in equation (3)), which is a direct effect of social support on HAZ or 224 BAZ after taking into account a mediation (indirect) effect of food security. Finally, average causal 225 mediation effects (ACME) are defined as the mean difference in effect between two counterfactual 226 states of a mediator, assuming no change in the initial condition (total effect subtracted by direct 227 effect : $\gamma-\gamma^{\prime}$, which equals to a product of the coefficient of $\beta$ in the equation (1) and $\delta$ in equation 
228 (3)). Mediation analysis was performed using use written code -medeff- in STATA $14 .^{30-32}$ 229 Analyses were performed separately by wave (age 8 and age 12) and country.

\section{Results}

The pattern of social supports greatly differed between settings (Fig 2). The average

232 number of maternal social supports was highest in Vietnam (3.26), followed by Ethiopia (2.40).

233 The average number of child supports was highest in India where $91.5 \%$ of children respondents

234 answered that they had someone to help in all six different kinds of situations. The relationship

235 between the level of maternal and child social support and average HAZ and BAZ was not 236 consistent across the countries. 


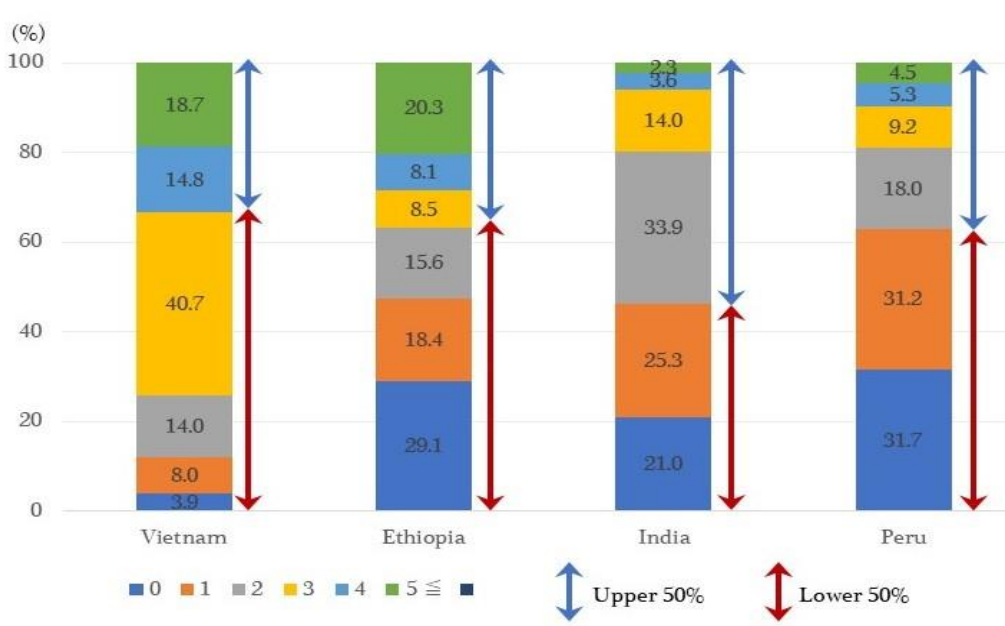

Fig 2.1 Distribution of maternal social support

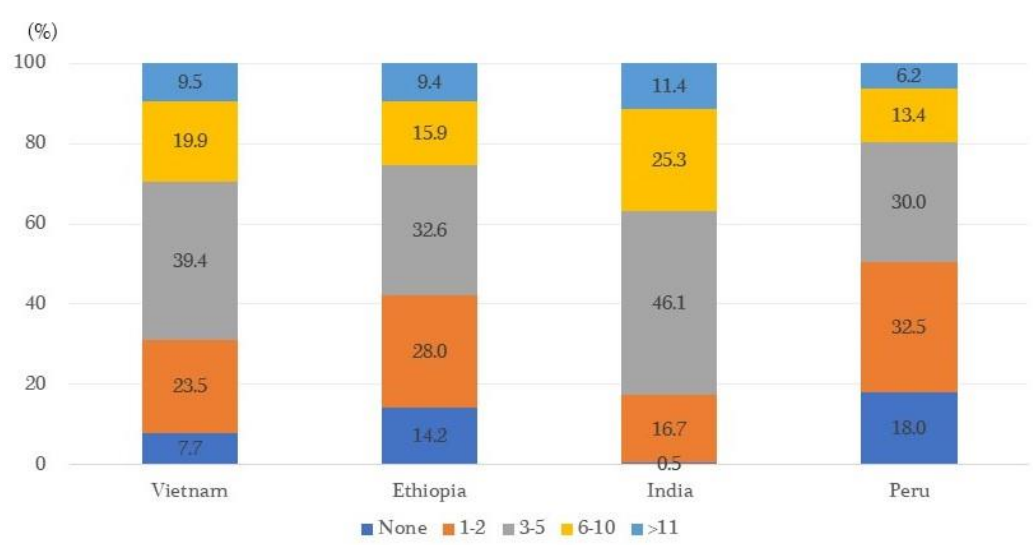

Fig 2.2 Distribution of maternal financial support

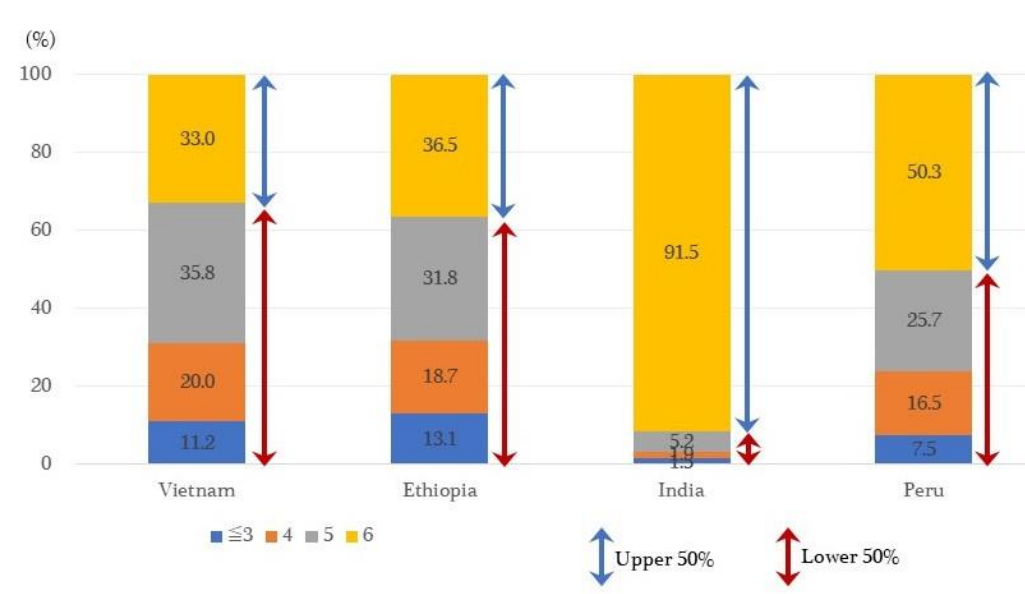

Fig 2.3 Distribution of child social support

\begin{tabular}{|c|c|c|c|}
\hline \multicolumn{2}{|c|}{} & L50\% & U50\% \\
\hline \multirow{4}{*}{ Vietnam } & Avg.SS & 2.38 & 4.96 \\
& zhfa & -1.47 & -1.45 \\
& zbfa & -1.15 & -1.09 \\
\hline \multirow{4}{*}{ Ethiopia } & Avg.SS & 0.73 & 5.26 \\
& zhfa & -1.46 & -1.56 \\
& zbfa & -1.25 & -1.32 \\
\hline \multirow{4}{*}{ Peru } & Avg.SS & 0.55 & 2.55 \\
& zhfa & -1.5 & -1.54 \\
& zbfa & -1.41 & -1.37 \\
\hline & Avg.SS & 0.50 & 2.96 \\
& zhfa & -1.35 & -1.46 \\
& zbfa & 0.45 & 0.56 \\
\hline
\end{tabular}

Avg.SS: Average number of maternal social supports

\begin{tabular}{|c|c|c|c|}
\hline \multicolumn{2}{|c|}{} & No & Yes \\
\hline \multirow{2}{*}{ Vietnam } & zhfa & -1.56 & -1.44 \\
& zbfa & -1 & -1.01 \\
\hline \multirow{2}{*}{ Ethiopia } & zhfa & -1.22 & -1.41 \\
& zbfa & -1.58 & -1.7 \\
\hline \multirow{2}{*}{ India } & zhfa & -1.31 & -1.53 \\
& zbfa & -2.55 & -1.46 \\
\hline \multirow{2}{*}{ Peru } & zhfa & -1.64 & -1.44 \\
& zbfa & 0.26 & 0.26 \\
\hline
\end{tabular}

\begin{tabular}{|c|c|c|c|}
\hline \multicolumn{2}{|c|}{} & L50\% & U50\% \\
\hline \multirow{4}{*}{ Vietnam } & Ave.SS & 4.3 & 6 \\
& zhfa & -1.48 & -1.34 \\
& zbfa & -1.01 & -1.04 \\
\hline \multirow{4}{*}{ Ethiopia } & Ave.SS & 4.1 & 6 \\
& zhfa & -1.34 & -1.44 \\
& zbfa & -1.67 & -1.71 \\
\hline \multirow{4}{*}{ Peru } & Ave.SS & 4.3 & 6 \\
& zhfa & -1.36 & -1.56 \\
& zbfa & -1.38 & -1.49 \\
\hline \multirow{2}{*}{ Ave.SS } & 4.3 & 6 \\
& zhfa & -1.53 & -1.42 \\
& zbfa & 0.26 & 0.26 \\
\hline
\end{tabular}

Avg.SS: Average number of child social supports

Figure 2. Distribution of social support 
Table S2 shows the descriptive statistics of the study samples at wave 1 from Vietnam,

248 Ethiopia, India, and Peru and the mean HAZ and BAZ for each category (characteristics of study

249 samples at wave 2 hardly changed from wave 1 because the Young Lives data is a cohort survey).

250 Generally, children with higher HAZ/BAZ were more likely to be from households that had fewer

251 household members, were wealthier and more likely to be in an urban area. The average BAZ for

252 the entire sample was remarkably high in Peru compared to other countries in both waves.

253 Table 1. Association between maternal and child support and child z-score for height at wave 1 and 2 in

254 four countries from linear regression adjusted for community cluster effect.

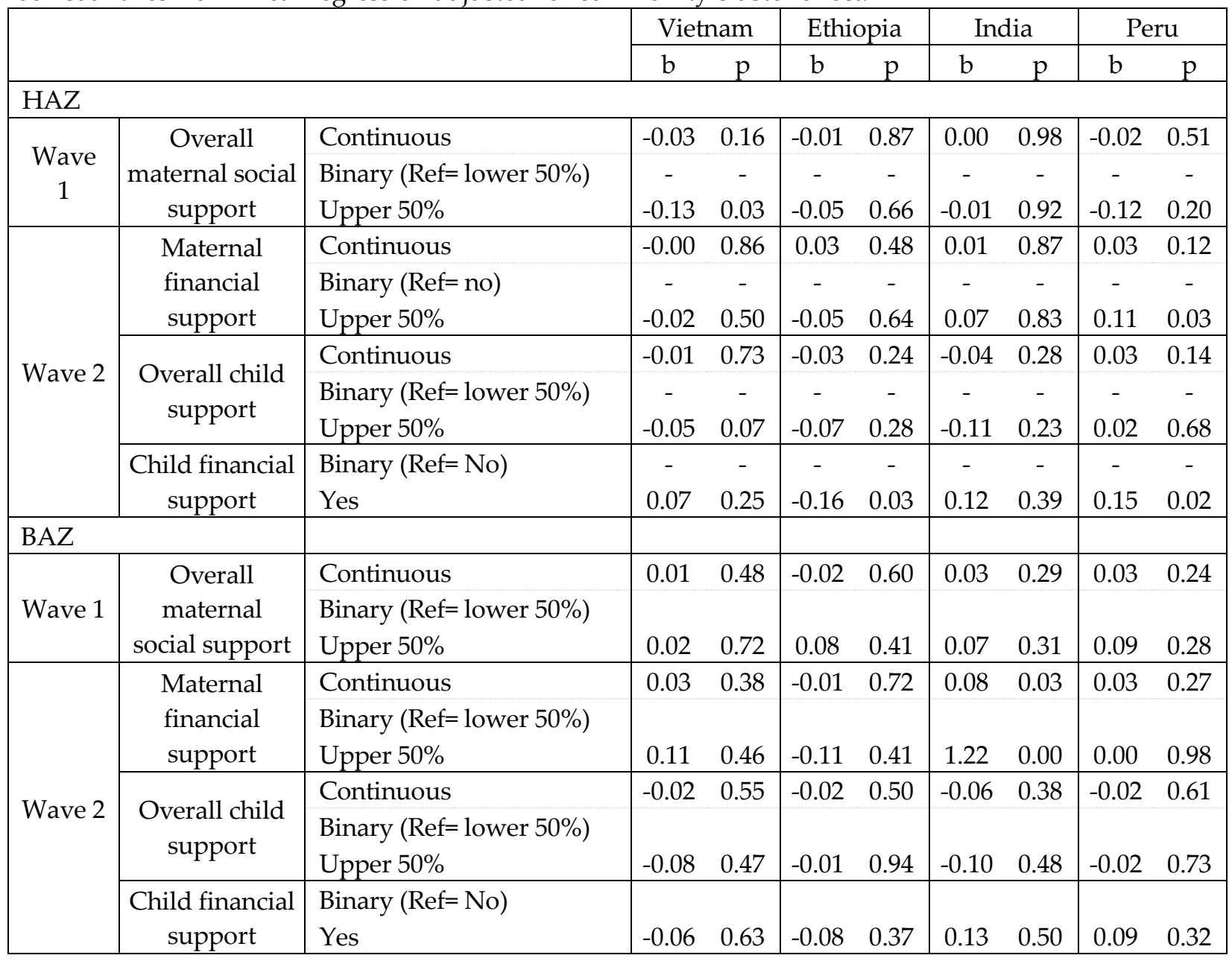


2571 and 2 were mixed (Table 1). At wave 1 when the child was 8 years old, children of mothers in

258 Vietnam whose overall level of social support belonged to the upper 50\% were likely to be lower 259 in HAZ, which was against our expectation. There was no significant association in the other three 260 countries. At wave 2, only Peru showed a positive association between the level of maternal 261 financial support and child's HAZ. Child financial support was negatively associated with HAZ 262 in Ethiopia while it was positively associated in Peru. The overall level of child support showed 263 no association in any countries. As for BAZ, only the level of maternal financial support, 264 operationalized both as a continuous and binary variable, showed a positive association when a 265 child is 12 years old in India (Table 1).

Table 2. Association between maternal and child social support and food security at waves 1 and 2 in four countries from linear regression adjusted for community cluster effect.

\begin{tabular}{|c|c|c|c|c|c|c|}
\hline & \multicolumn{2}{|c|}{$\begin{array}{c}\text { Maternal support at } \\
\text { wave 1 }\end{array}$} & \multicolumn{2}{c|}{$\begin{array}{c}\text { Child financial support at } \\
\text { wave 2 }\end{array}$} & \multicolumn{2}{c|}{$\begin{array}{c}\text { Maternal financial support at } \\
\text { wave 2 }\end{array}$} \\
\cline { 2 - 7 } & Ref & Upper 50\% & Ref & Upper 50\% & Ref & Upper 50\% \\
\hline Vietnam & - & $4.75(0.60-37.46)$ & & & & \\
Ethiopia & & & - & $0.88(0.65-1.20)$ & & \\
India & & & - & & - & $4.44(0.59-33.19)$ \\
Peru & & & & $0.87(0.48-1.59)$ & - & $1.66(1.07-2.60)$ \\
\hline
\end{tabular}

Table 2 shows results from the linear regression models examining the association

270 between social support and food security. As previously described in the Methods section, we

271 limited the analyses only to the social support variables that showed significant associations with

272 HAZ or BAZ (presented in Table 2). Only the level of maternal financial support in Peru was

273 significantly positively associated with probability of having food security at wave 2. 
274 Table 3. Mediated effect of maternal financial support on child's HAZ in Peru via securing enough food.

\begin{tabular}{|l|c|c|c|c|}
\hline & ACME & ADE & Total effect & $\%$ of total effect mediated \\
\hline HAZ at & \multicolumn{4}{|c|}{ Upper $50 \%$ vs. lower $50 \%$ of maternal financial support via enough food in Peru } \\
\cline { 2 - 6 } wave 2 & $-0.01(-0.03,0.01)$ & $0.12(0.01,0.22)$ & $0.11(0.01,0.21)$ & $-0.08(-0.42,-0.04)$ \\
\hline
\end{tabular}

Finally, causal mediation analysis using Imai et al's method was performed only in Peru,

277 since it was the only country to show a significant association between maternal financial support

278 and food security (Table 3). Our model to test the mediating role of food security in linking

279 maternal financial social support with a child's HAZ in Peru showed that the ACME of upper 50\%

280 of maternal financial support is less than zero and statistically non-significant at $95 \%$ level,

281 implying that role of food security is not a significant mediator of the impact of maternal financial

282 support on child's height.

283 Discussion

284 Although there has been much effort to elucidate whether social capital has any beneficial

285 effect on a child's nutritional status, results on the effect of maternal social capital have been mixed

286 across studies depending on the types of social capital, child's age, and global setting. Also,

287 improved food security has been hypothesized as one of the key mechanisms to explain the

288 positive effect of social capital on child anthropometry, but it has never been examined empirically

289 to our knowledge.

290 Limitations

291 There are several limitations to consider when interpreting the results. First, although the

292 YL study is a cohort survey, we could not exploit the longitudinal design for the analyses because 
293 social capital was not uniformly measured across the waves. Cross-sectional analysis limits our

294 ability to draw causal inferences. Second, the level of maternal and child social support was

295 arbitrarily categorized. We classified the level of maternal and child social support as being in the

296 upper or lower 50\% using median cutoff values, which was our decision to maintain a consistent

297 standard across countries because the distribution of social support differed substantially by

298 country. However, to reduce the possibility of bias from arbitrary operationalization of the

299 variables, we presented results from both models wherein social support was operationalized as

300 both a continuous and a binary variable. Finally, the data for the study is more than 15 years old,

301 which may raise the question of whether the results remain valid under the current context.

302 However, the findings of our study still may offer implications to other LMIC currently

303 undergoing similar contexts of the study countries in the survey years.

\section{$304 \quad$ Interpretation of findings}

Although social capital has been demonstrated to have a beneficial effect on a range of

306 health outcomes, especially on mental health, the effect of maternal social capital on child

307 anthropometry have been inconsistent. Our results did not support that maternal and child

308 support are strongly or consistently associated with a child's nutritional status.

In De Silva's study (2007), the significance of associations between maternal social support

310 and height or weight of children aged between 6 and 18 months varied across four LMICs.

311 Significant associations with a child's height were found between the level of maternal social 312 support in Peru, Vietnam, and Ethiopia. As for child's weight, a significant association was found 313 only in Vietnam. However, unlike our results, the direction was consistently positive, which is 
314 assumed to be due to the difference in the age of the study population. Our analyses targeted

315 children aged 8 years old (wave 1) and 12 years old (wave 2) which is much older compared to 316 the sample in De Silva's analysis (2007). Height and weight at a younger age are more sensitive to 317 feeding status or growth stimulation than in the later stage of growth. Evidence shows that catch318 up growth of preterm infants measured by weight or length mainly occurred from the $10^{\text {th }}$ to $12^{\text {th }}$ 319 month of their lives. ${ }^{33}$ Another study reported that the catch-up growth of malnutrition of 320 institutionalized children who were adopted before the age of 12 months was much larger than 321 the children adopted after 12 months. ${ }^{34}$ Any effect of maternal social support on child's height or 322 BMI are therefore likely to be more pronounced among younger children.

324 on the child's nutritional status. Social support enables mothers to access knowledge (e.g., how to 325 feed their child for better nutritional status), and to give better care (e.g., practicing hygiene habits 326 or breastfeeding for longer). ${ }^{35}$ This effect would be more marked in societies where mothers have 327 a lower background level of education, and therefore, could not have obtained the necessary 328 knowledge through schooling. Emotional support is beneficial for maternal mental health, which 329 also can be linked to improved child growth. ${ }^{3637}$ Martin et al (2004) provided another theory that 330 social capital is associated with reduced odds of household hunger and food insecurity. ${ }^{16}$ 331 Availability and access to food can be enhanced by collectively sharing information and resources. 332 In a developing country context, sharing seeds and livestock breeds can be one of the examples. 333 Further, in communities with strong ties, solidarity, and networks, people can share the food itself 334 during times of hunger. ${ }^{19}$ However, our analyses revealed that the child's nutritional status was 
335 associated only with financial support both for mother and child, and food security was not a 336 mediator.

There are several possible explanations for the lack of mediation by food insecurity. First,

338 the YL study over-sampled poor sites, and the data from India were collected only in the state of 339 Andhra Pradesh which is one of the poorest states. Therefore, food would not have been sufficient 340 across the community. Even if someone had social supports these sources of supports might have 341 not had enough food to share. In Cattel's (2001) qualitative study, individuals who are part of 342 homogenous networks made up of poor people are less likely to receive effective support because 343 other members are also not able to provide the required assistance. ${ }^{15} 38$ Second, since the 344 improvement of child anthropometry requires a continuous supply of a well-balanced diet, one345 off or sporadic type of support would not be linked to improving child anthropometry. Cultural 346 specificity in a social network may determine to what extent and how people are able to give and 347 take support from each other. Therefore, having someone to rely on in specific conditions may or 348 may not mean long-term and stable support depending on the context. ${ }^{15}$ Questions about the 349 strength of ties with the source of supports or frequency of receiving help from them would help 350 uncover the practical contribution of social support to a child's nutritional status. Our findings suggest that interventions to strengthen social support in anticipation of a 352 positive effect on improving child's nutritional status may be unreliable in very poor communities. 353 Also, considering the between-country variability implies that a "one fits all" approach for 354 enhancing social capital may not be appropriate. 
356 whether boosting maternal or child social support can be a practical means to improving a child's

357 height and BMI in a resource-poor setting. Future research needs to repeat the current analysis 358 using more sophisticated measurements of social support (i.e., measuring strength and frequency 359 of support) and based on a more recent dataset with a larger sample size to confirm the findings.

361 Conclusion

Strengthening social support within homogenously resource-poor setting may not be a 363 ideal intervention to improve child nutritional status because sources of supports may lack 364 sufficient food resources to share. Considering between-country heterogeneity in association 365 between social support and child nutritional status, a "one size fits all" approach for enhancing 366 social capital may not be appropriate.

368 Ethical Approval and consent to participate.

369 Ethical approval was not required as Young Lives Study provides anonymous, secondary data that is 370 publicly available for scientific use.

\section{Consent for publication}

372 Not applicable

\section{Availability of data and materials}

374 Data are available from the UK Data Service website (at 375 https://discover.ukdataservice.ac.uk/series/?sn=2000060). Users are required to register 
376 and apply for a password with the UK Data Service and sign a confidentiality agreement

377 before getting access to the data. Also, users are asked to inform the UK Data Service and

378 Young Lives of analysis or publication resulting from their work with the dataset.

379 Competing interests

380 The authors declare no conflict.

381 Funding

382 There was no funding to support this study.

383 Authors' contribution

384 HYL conceived the idea. HYL, HIS, and IK designed the study. HYL analyzed the data 385 and wrote the first draft. All authors interpreted the results. IHS and IK revised the 386 manuscript critically and supervised the whole process. All authors read and approved 387 the final manuscript.

388 Acknowledgements

389 Not applicable

390 Author details

391 'Department of Global Health and Population, Harvard T.H.Chan School of Public Health, 392 Boston, MA, USA. 2 Institute of Convergence Science (ICONS), Convergence Science Academy, 393 Yonsei University, Seoul, The Republic of Korea ${ }^{3}$ Graduate School of Social Welfare, Yonsei 394 University, Seoul, The Republic of Korea ${ }^{4}$ Department of Social and Behavioral Sciences, Harvard 395 T.H. Chan School of Public Health, Boston, MA, USA 
396

397

398

399

400

401

402

403

404

405

406

407

408

409

410

411

412

413

414

415

416

417

418

419

420

421

422

423

424

425

426

427

428 


\section{References}

1. Blossner M, De Onis M, Pru“ss-U"stu"n A. Malnutrition: quantifying the health impact at national and local levels: World Health Organization 2005.

2. Miller AC, Murray MB, Thomson DR, et al. How consistent are associations between stunting and child development? Evidence from a meta-analysis of associations between stunting

3. Sudfeld CR, McCoy DC, Danaei G, et al. Linear growth and child development in low-and middle-income countries: a meta-analysis. J Pediatrics 2015;135(5):e1266-e75.

4. Black RE, Victora CG, Walker SP, et al. Maternal and child undernutrition and overweight in low-income and middle-income countries. The lancet 2013;382(9890):427-51.

5. UNICEF/WHO/WB. UNICEF/WHO/World Bank joint child malnutrition estimates: global and regional Accessed through : https://data.unicef.org/topic/nutrition/malnutrition/. 2020

6. UNICEF. mproving child nutrition: the achievable imperative for global progress (Accessed March 24, 2020. https://www.unicef.org/nutrition/index 68661.html). : United Nations Children's Fund, 2020.

7. Debbie L Humphries, Kirk A Dearden, Benjamin T Crookston,, Lia C Fernald, Aryeh D Stein, Tassew Woldehanna, Mary E Penny, and Jere R Behrman, on behalf of The Young Lives Determinants and Consequences of Child Growth Project Team. Cross-sectional and

453 8. Dubos R. Social capital: Theory and research: Routledge 2017.

454 9. Lin N. Building a network theory of social capital. Connections 1999;22(1):28-51. 
10. Forsman AK, Nyqvist F, Schierenbeck I, et al. Structural and cognitive social capital and depression among older adults in two Nordic regions. Aging \& mental health 2012;16(6):771-79.

11. Watson GW, Papamarcos SD. Social capital and organizational commitment. Journal of business psychology 2002;16(4):537-52.

12. Vikram K. Social capital and child nutrition in India: The moderating role of development. Health \& place 2018;50:42-51.

13. Sujarwoto S, Tampubolon G. Mother's social capital and child health in Indonesia. Social Science \& Medicine 2013;91:1-9.

14. Harpham T, De Silva MJ, Tuan T. Maternal social capital and child health in Vietnam. Journal of Epidemiology Community Health 2006;60(10):865-71.

15. De Silva MJ, Harpham T. Maternal social capital and child nutritional status in four developing countries. Health \& place 2007;13(2):341-55.

16. Martin KS, Rogers BL, Cook JT, et al. Social capital is associated with decreased risk of hunger. Social science \& medicine 2004;58(12):2645-54.

17. Walker JL, Holben DH, Kropf ML, et al. Household food insecurity is inversely associated with social capital and health in females from special supplemental nutrition program for women, infants, and children households in Appalachian Ohio. Journal of the American Dietetic Association 2007;107(11):1989-93.

18. Dean WR, Sharkey JR, Johnson CM. Food insecurity is associated with social capital, perceived personal disparity, and partnership status among older and senior adults in a largely rural area of central Texas. Journal of nutrition in gerontology geriatrics 2011;30(2):169-86.

19. Sseguya H, Mazur RE, Flora CB. Social capital dimensions in household food security interventions: implications for rural Uganda. Agriculture Human values 2018;35(1):117-29.

20. Barnett I, Ariana P, Petrou S, et al. Cohort profile: the Young Lives study. J International journal of epidemiology 2013;42(3):701-08.

21. group. Ys. Young lives methods guide: sampling (Available: http://www.younglives.org.uk/files/methods-guide/methods-guide-sampling). 
22. Bailey K, Ferro-Luzzi A. Use of body mass index of adults in assessing individual and community nutritional status. Bulletin of the World Health Organization 1995;73(5):673.

23. Onis Md, Onyango AW, Borghi E, et al. Development of a WHO growth reference for schoolaged children and adolescents. Bulletin of the World health Organization 2007;85:660-67.

24. WHO. WHO child growth standards: training course on child growth assessment. 2008

25. UN. How to weigh and measure children: assessing the nutritional status of young children in household surveys. New York.: UN. , 1986.

26. WHO. WHO reference 2007 STATA macro package.

27. Baron RM, Kenny DA. The moderator-mediator variable distinction in social psychological research: Conceptual, strategic, and statistical considerations. Journal of personality social psychology 1986;51(6):1173.

28. Imai K, Keele L, Tingley D. A general approach to causal mediation analysis. Psychological methods 2010;15(4):309.

29. Imai K, Keele L, Yamamoto T. Identification, inference and sensitivity analysis for causal mediation effects. Statistical science 2010:51-71.

30. Hicks R, Tingley D. Causal mediation analysis. The Stata Journal 2011;11(4):605-19.

31. MacKinnon DP, Lockwood CM, Williams J. Confidence limits for the indirect effect: Distribution of the product and resampling methods. Multivariate behavioral research 2004;39(1):99-128.

32. MacKinnon DP, Fairchild AJ, Fritz MS. Mediation analysis. Annu Rev Psychol 2007;58:593-614.

33. Gong Y-H, Ji C-Y, Shan J-P. A longitudinal study on the catch-up growth of preterm and term infants of low, appropriate, and high birth weight. Asia Pacific Journal of Public Health 2015;27(2):NP1421-NP31.

34. Van IJzendoorn MH, Bakermans-Kranenburg MJ, Juffer F. Plasticity of growth in height, weight, and head circumference: meta-analytic evidence of massive catch-up after international adoption. Journal of Developmental Behavioral Pediatrics 2007;28(4):334-43. 
509 35. Anderson AK, Damio G, Himmelgreen DA, et al. Social capital, acculturation, and 510 breastfeeding initiation among Puerto Rican women in the United States. Journal of Human $511 \quad$ Lactation 2004;20(1):39-45.

512 36. Patel V, Rahman A, Jacob K, et al. Effect of maternal mental health on infant growth in low 513 income countries: new evidence from South Asia. BMJ 2004;328(7443):820-23.

514 37. Harpham T, Huttly S, De Silva MJ, et al. Maternal mental health and child nutritional status in 515 four developing countries. Journal of Epidemiology Community Health 2005;59(12):1060-64.

516 38. Cattell V. Poor people, poor places, and poor health: the mediating role of social networks and 517 social capital. Social science E medicine 2001;52(10):1501-16. 
Figures

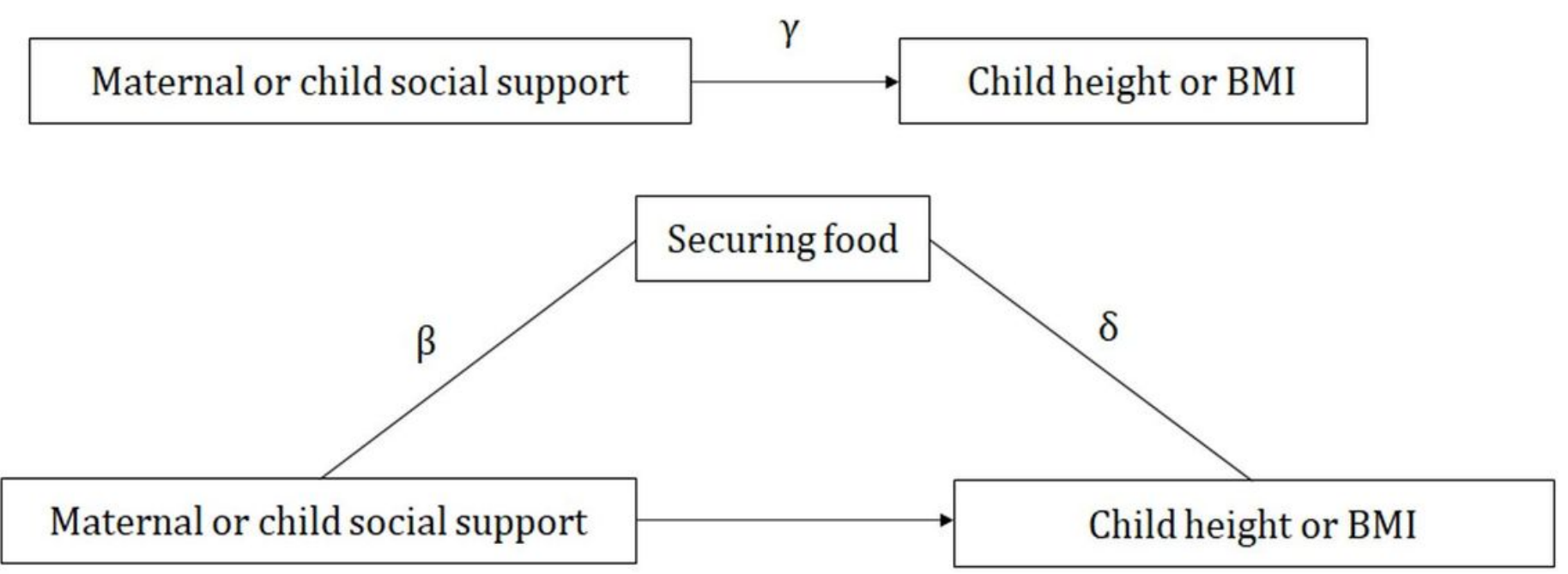

\section{Figure 1}

The mediation model. 


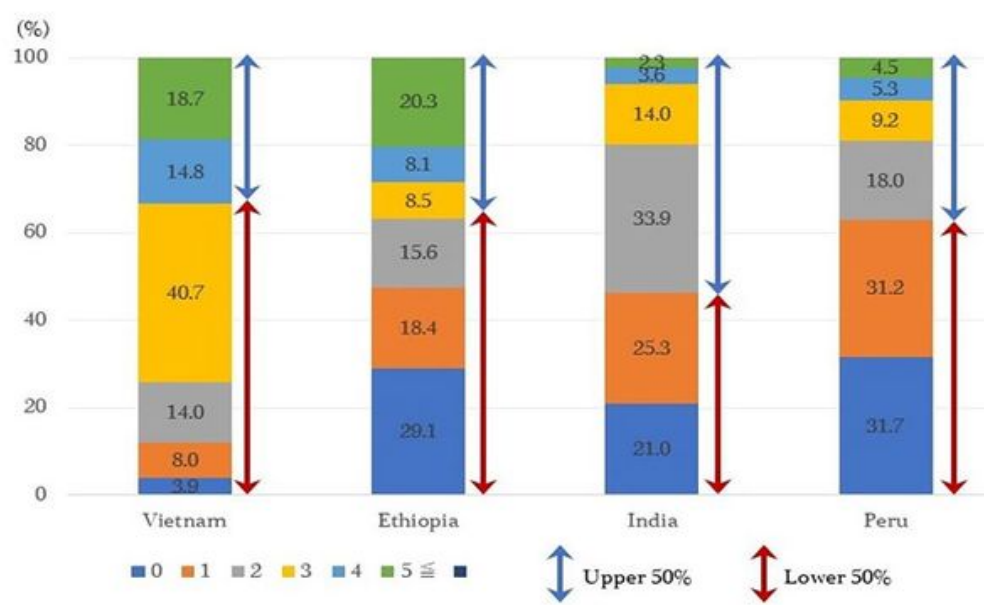

Fig 2.1 Distribution of maternal social support

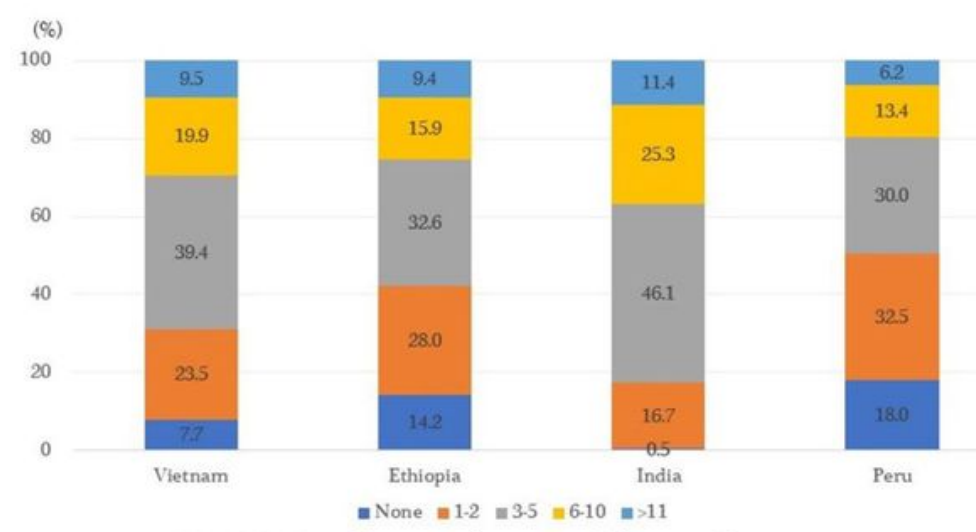

Fig 2.2 Distribution of maternal financial support

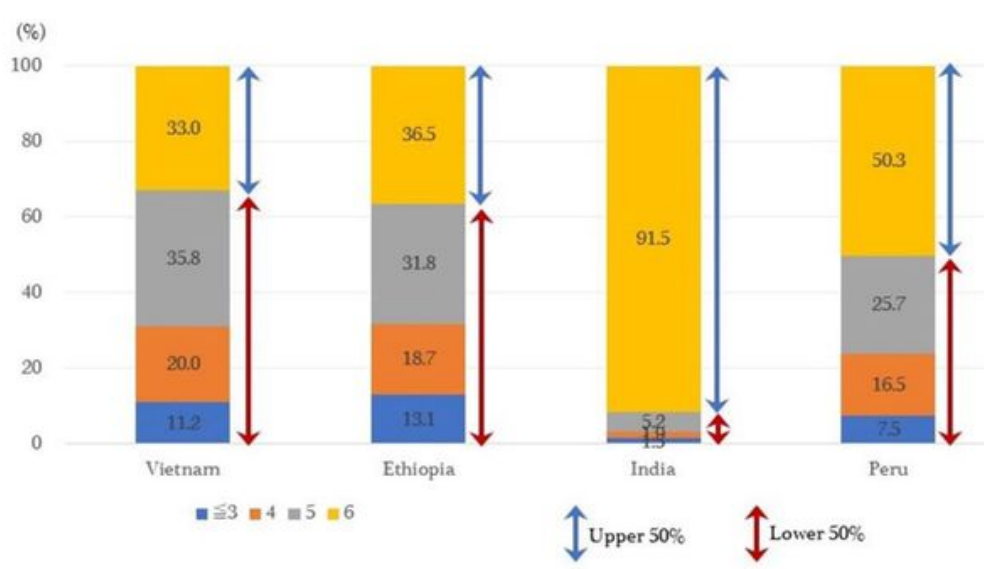

Fig 2.3 Distribution of child social support

\begin{tabular}{|c|c|c|c|}
\hline \multicolumn{2}{|c|}{} & L50\% & U50\% \\
\hline \multirow{4}{*}{ Vietnam } & Avg.SS & 2.38 & 4.96 \\
& zhfa & -1.47 & -1.45 \\
& zbfa & -1.15 & -1.09 \\
\hline \multirow{4}{*}{ Ethiopia } & Avg.SS & 0.73 & 5.26 \\
& zhfa & -1.46 & -1.56 \\
& zbfa & -1.25 & -1.32 \\
\hline \multirow{4}{*}{ India } & Avg.SS & 0.55 & 2.55 \\
& zhfa & -1.5 & -1.54 \\
& zbfa & -1.41 & -1.37 \\
\hline \multirow{4}{*}{ Peru } & Avg.SS & 0.50 & 2.96 \\
& zhfa & -1.35 & -1.46 \\
& zbfa & 0.45 & 0.56 \\
\hline
\end{tabular}

Avg.SS: Average number of maternal social supports

\begin{tabular}{|c|c|c|c|}
\hline \multicolumn{2}{|c|}{} & No & Yes \\
\hline \multirow{2}{*}{ Vietnam } & zhfa & -1.56 & -1.44 \\
& zbfa & -1 & -1.01 \\
\hline \multirow{2}{*}{ Ethiopia } & zhfa & -1.22 & -1.41 \\
& zbfa & -1.58 & -1.7 \\
\hline \multirow{2}{*}{ India } & zhfa & -1.31 & -1.53 \\
& zbfa & -2.55 & -1.46 \\
\hline \multirow{2}{*}{ Peru } & zhfa & -1.64 & -1.44 \\
& zbfa & 0.26 & 0.26 \\
\hline
\end{tabular}

\begin{tabular}{|c|c|c|c|}
\hline \multicolumn{2}{|c|}{} & L50\% & U50\% \\
\hline \multirow{4}{*}{ Vietnam } & Ave.SS & 4.3 & 6 \\
& zhfa & -1.48 & -1.34 \\
& zbfa & -1.01 & -1.04 \\
\hline \multirow{4}{*}{ Ethiopia } & Ave.SS & 4.1 & 6 \\
& zhfa & -1.34 & -1.44 \\
& zbfa & -1.67 & -1.71 \\
\hline \multirow{4}{*}{ India } & Ave.SS & 4.3 & 6 \\
& zhfa & -1.36 & -1.56 \\
& zbfa & -1.38 & -1.49 \\
\hline \multirow{4}{*}{ Peru } & Ave.SS & 4.3 & 6 \\
& zhfa & -1.53 & -1.42 \\
& zbfa & 0.26 & 0.26 \\
\hline
\end{tabular}

Avg.SS: Average number of child social supports

\section{Figure 2}

Distribution of social support

\section{Supplementary Files}

This is a list of supplementary files associated with this preprint. Click to download. 
- SupplementalNJ.docx 